\begin{tabular}{|c|l|}
\hline Title & Global properties of spacelike curves in Minkowski 3-space \\
\hline Author(s) & Izumiya, Shyuichi; Kikuchi, Makoto; Takahashi, Masatomo \\
\hline Citation & Hokkaido University Preprint Series in Mathematics, 713, 1-12 \\
\hline Issue Date & 2005 \\
\hline DOI & 10.14943/83864 \\
\hline Doc URL & http://hdl.handle.net/2115/69518 \\
\hline Type & bulletin (article) \\
\hline File Information & pre713.pdf \\
\hline
\end{tabular}

Instructions for use 


\title{
Global properties of spacelike curves in Minkowski 3-space
}

\author{
Shyuichi IZUMIYA, Makoto KIKUCHI and Masatomo TAKAHASHI
}

April 6, 2005

\begin{abstract}
We study global properties of closed spacelike curves in Minkowski 3-space.
\end{abstract}

\section{Introduction}

Recently there appeared some articles on differential geometry of spacelike submanifolds in Minkowski space [2, 3, 4, 5, 7, 8]. A natural question is how global properties of spacelike submanifolds in Minkowski space are different from those properties of submanifold in Euclidean space? The simplest case is spacelike curves in Minkowski 3-space. In this paper we study global properties of spacelike closed curves in Minkowski 3-space. There exists the canonical Euclidean plane in Minkowski 3-space with the natural projection from Minkowski 3-space (cf., §2). If we consider a regular (immersed) closed curve in such the plane, then it is a spacelike regular closed curve.

Firstly we consider a problem how global properties of spacelike closed curves are different from those of closed Euclidean plane curves. For any regular spacelike curve, the projection image is a regular plane curve. Therefore the rotation number of the projection image is a regular homotopy invariant for spacelike regular closed curves. The converse assertion is also true for regular spacelike closed curves (cf., Theorem 3.1). It follows from the theorem of Whitney [12] that the rotation number of the projection image of a spacelike regular closed curve is a complete invariant with respect to the spacelike regular homotopy. We also give a Lorentzian geometric formula for calculating the rotation number of the projection image of a spacelike regular closed curve (cf., Theorem 3.8).

Secondary, we consider spacelike knots (closed embedded spacelike curves). In order to avoid wildness we consider in the PL-category. We consider the spacelike isotopy among spacelike knots and show that two spacelike knots $K$ and $K^{\prime}$ are spacelike isotopic if and only if these are isotopic (as the ordinary sense) and the regular projection images (Minkowski spacelike regular diagrams) of these spacelike knots are regular isotopic. Here we say that two regular diagrams in a plane is regular isotopic if we can change one of the regular diagram to another regular

2000 Mathematics Subject classification.

Key Words and Phrases. closed spacelike curve, regular isotopy

partially supported by Grant-in-Aid for formation of COE 
diagram by performing, a finite number of times, the Reidemeister moves $\Omega_{2}, \Omega_{3}$ (cf., Fig. 1 in $\S 4)$. In [10], the oriented regular isotopic class of oriented regular diagrams given by the same oriented knot up to isotopy (in the sense of the ordinary knot theory) is completely determined by the pair of the rotation number and the writhe of the regular diagrams. It follows that the spacelike isotopy among spacelike knots is completely determined by the pair of the rotation number and the writhe of the regular projection images up to the ordinary isotopy.

\section{$2 \quad$ Basic facts and notations}

In this section we prepare some basic notions on Minkowski 3-space and spacelike curves. For basic concepts and properties, see [11]. Let $\mathbb{R}^{3}=\left\{\left(x_{0}, x_{1}, x_{2}\right) \mid x_{i} \in \mathbb{R}(i=0,1,2)\right\}$ be a 3-dimensional cartesian space. For any $\boldsymbol{x}=\left(x_{0}, x_{1}, x_{2}\right), \boldsymbol{y}=\left(y_{0}, y_{1}, y_{2}\right) \in \mathbb{R}^{3}$, the pseudo scalar product of $\boldsymbol{x}$ and $\boldsymbol{y}$ is defined by

$$
\langle\boldsymbol{x}, \boldsymbol{y}\rangle=-x_{0} y_{0}+x_{1} y_{1}+x_{2} y_{2} .
$$

We call $\left(\mathbb{R}^{3},\langle\rangle,\right)$ Minkowski 3 -space. We denote $\mathbb{R}_{1}^{3}$ instead of $\left(\mathbb{R}^{3},\langle\rangle,\right)$. We say that a nonzero vector $\boldsymbol{x} \in \mathbb{R}_{1}^{3}$ is spacelike, lightlike or timelike if $\langle\boldsymbol{x}, \boldsymbol{x}\rangle>0,\langle\boldsymbol{x}, \boldsymbol{x}\rangle=0$ or $\langle\boldsymbol{x}, \boldsymbol{x}\rangle<0$ respectively. The norm of the vector $\boldsymbol{x} \in \mathbb{R}_{1}^{3}$ is defined by $\|\boldsymbol{x}\|=\sqrt{|\langle\boldsymbol{x}, \boldsymbol{x}\rangle|}$. We have the canonical projection $\pi: \mathbb{R}_{1}^{3} \longrightarrow \mathbb{R}^{2}$ defined by $\pi\left(x_{0}, x_{1}, x_{2}\right)=\left(x_{1}, x_{2}\right)$. Here we identify $\{\mathbf{0}\} \times \mathbb{R}^{2}$ with $\mathbb{R}^{2}$ and it is considered as Euclidean plane whose scalar product is induced from the pseudo scalar product $\langle$,$\rangle . We denote that$

$$
L C_{+}^{*}=\left\{\boldsymbol{x}=\left(x_{0}, x_{1}, x_{2}, x_{3}\right) \in L C_{0} \mid x_{0}>0,\langle\boldsymbol{x}, \boldsymbol{x}\rangle=0\right\}
$$

and we call it the future lightcone at the origin. If $\boldsymbol{x}=\left(x_{0}, x_{1}, x_{2}\right)$ is a lightlike vector, then $x_{0} \neq 0$. Therefore we have

$$
\widetilde{\boldsymbol{x}}=\left(1, \frac{x_{1}}{x_{0}}, \frac{x_{2}}{x_{0}}\right) \in S_{+}^{1}=\left\{\boldsymbol{x}=\left(x_{0}, x_{1}, x_{2}\right) \mid\langle\boldsymbol{x}, \boldsymbol{x}\rangle=0, x_{0}=1\right\} .
$$

We call $S_{+}^{1}$ the lightcone (or, spacelike) unit circle.

For any $\boldsymbol{x}=\left(x_{0}, x_{1}, x_{2}\right), \boldsymbol{y}=\left(y_{0}, y_{1}, y_{2}\right) \in \mathbb{R}_{1}^{3}$, the pseudo vector product of $\boldsymbol{x}$ and $\boldsymbol{y}$ is defined as follows:

$$
\boldsymbol{x} \wedge \boldsymbol{y}=\left|\begin{array}{ccc}
-\boldsymbol{e}_{0} & \boldsymbol{e}_{1} & \boldsymbol{e}_{2} \\
x_{0} & x_{1} & x_{2} \\
y_{0} & y_{1} & y_{2}
\end{array}\right|=\left(-\left(x_{1} y_{2}-x_{2} y_{1}\right), x_{2} y_{0}-x_{0} y_{2}, x_{0} y_{1}-x_{1} y_{0}\right)
$$

where $\left\{\boldsymbol{e}_{0}, \boldsymbol{e}_{1}, \boldsymbol{e}_{2}\right\}$ is the canonical basis of $\mathbb{R}_{1}^{3}$.

We now review Lorentzian differential geometry on spacelike regular curves. Let $\gamma: I \longrightarrow \mathbb{R}_{1}^{3}$ be a spacelike regular curve, where $I$ is an open interval. The arc-length of a spacelike curve $\gamma$, measured from $\gamma\left(t_{0}\right), t_{0} \in I$ is

$$
s(t)=\int_{t_{0}}^{t}\|\dot{\gamma}(t)\| d t
$$

where $\dot{\gamma}=d \gamma / d t$. Then a parameter $s$ is determined such that $\left\|\gamma^{\prime}(s)\right\|=1$, where $\gamma^{\prime}(s)=d \gamma / d s$. Consequently we say that a spacelike regular curve $\gamma$ is parameterized by arc-length if it satisfies 
$\left\|\gamma^{\prime}(s)\right\|=1$. We now assume that the parameter $s$ of $\gamma$ is the arc-length parameter. Let us denote $\boldsymbol{t}(s)=\gamma^{\prime}(s)$, and we call $\boldsymbol{t}(s)$ a unit tangent vector of $\gamma$ at $s$. We define the curvature by $\kappa(s)=\left\|\gamma^{\prime \prime}(s)\right\|$. If $\kappa(s) \neq 0$, then the unit principal normal vector $\boldsymbol{n}(s)$ of the curve $\gamma$ at $s$ is given by $\gamma^{\prime \prime}(s)=\kappa(s) \boldsymbol{n}(s)$. The signature of $\boldsymbol{x}$ is defined to be

$$
\operatorname{sign}(\boldsymbol{x})=\left\{\begin{array}{rl}
1 & \boldsymbol{x} \text { is spacelike } \\
0 & \boldsymbol{x} \text { is lightlike } \\
-1 & \boldsymbol{x} \text { is timelike }
\end{array}\right.
$$

We write $\delta(\gamma(s))=\operatorname{sign}(\boldsymbol{n}(s))$. The unit vector $\boldsymbol{b}(s)=\boldsymbol{t}(s) \wedge \boldsymbol{n}(s)$ is called a unit binormal vector of the curve $\gamma$ at $s$. Since $\boldsymbol{t}(s)$ is spacelike, we have $\langle\boldsymbol{b}(s), \boldsymbol{b}(s)\rangle=-\delta(\gamma(s))$. Then the following Frenet-Serret type formula holds:

$$
\left\{\begin{aligned}
\boldsymbol{t}^{\prime}(s) & =\kappa(s) \boldsymbol{n}(s) \\
\boldsymbol{n}^{\prime}(s) & =-\delta(\gamma(s)) \kappa(s) \boldsymbol{t}(s)+\tau(s) \boldsymbol{b}(s), \\
\boldsymbol{b}^{\prime}(s) & =\tau(s) \boldsymbol{n}(s)
\end{aligned}\right.
$$

where $\tau(s)$ is the torsion of the curve $\gamma$ at $s$ (cf. [1]). This is the system of natural equations for the study of spacelike curves in $\mathbb{R}_{1}^{3}$. It is, however, useless at the point $\gamma(s)$ with $\kappa(s)=0$. We denote $\boldsymbol{N}(s)=\gamma^{\prime \prime}(s)$ and $\boldsymbol{B}(s)=\boldsymbol{t}(s) \wedge \boldsymbol{N}(s)$ under the assumption that $\gamma^{\prime \prime}(s) \neq 0$. We simply call $\boldsymbol{N}(s)$ a principal normal vector and $\boldsymbol{B}(s)$ a binormal vector. If $\kappa(s) \neq 0$, then we have $\boldsymbol{N}(s)=\kappa(s) \boldsymbol{n}(s)$ and $\boldsymbol{B}(s)=\kappa(s) \boldsymbol{b}(s)$. It follows that

$$
\langle\boldsymbol{N}(s) \pm \boldsymbol{B}(s), \boldsymbol{N}(s) \pm \boldsymbol{B}(s)\rangle=\kappa^{2}(s)(\delta(\gamma(s))-\delta(\gamma(s)))=0 .
$$

If $\kappa(s)=0$, then $\boldsymbol{N}(s)$ is a lightlike vector, so that any pseudo orthogonal vector in the normal plane of $\gamma(s)$ is parallel to $\boldsymbol{N}(s)$. This means that $\boldsymbol{N}(s) \pm \boldsymbol{B}(s)$ is a lightlike vector which is parallel to the vector $\boldsymbol{N}(s)$ for $s \in I$ with $\kappa(s)=0$.

For any spacelike regular curve $\gamma: I \longrightarrow \mathbb{R}_{1}^{3}$ with the arc-length parameter $s$, we define the lightcone Gauss map $\widetilde{\mathbb{L}}: I \longrightarrow S_{+}^{1}$ by

$$
\widetilde{\mathbb{L}}(s)=\boldsymbol{N}(\widetilde{s)+\boldsymbol{B}}(s) .
$$

By the previous arguments, we have $\widetilde{\mathbb{L}}(s)=\boldsymbol{n}(\widetilde{s)+\boldsymbol{b}}(s)$ when $\kappa(s) \neq 0$. Therefore we define a map $\mathbb{L}: I \longrightarrow L C^{*}$ by

$$
\mathbb{L}(s)=\boldsymbol{n}(s)+\boldsymbol{b}(s)
$$

under the condition that $\kappa(s) \neq 0$. In this case, we write that $\mathbb{L}(s)=\left(\ell_{0}(s), \ell_{1}(s), \ell_{2}(s)\right)$.

For a spacelike curve $\gamma: I \longrightarrow \mathbb{R}_{1}^{3}$, we now define a family of functions $H: I \times S_{+}^{1} \longrightarrow \mathbb{R}$ by $H(s, \boldsymbol{v})=\langle\gamma(s), \boldsymbol{v}\rangle$. We call $H$ a lightcone height function on $\gamma$. We denote $h(s)=H\left(s, \boldsymbol{v}_{0}\right)$ for $\boldsymbol{v}_{0} \in S_{+}^{1}$. In [2], we have shown the following proposition:

Proposition 2.1 Let $\gamma: I \longrightarrow \mathbb{R}_{1}^{3}$ be a unit sped spacelike curve with $\gamma^{\prime \prime}(s) \neq 0$.

(1) $h^{\prime}\left(s_{0}\right)=0$ if and only if $\boldsymbol{v}_{0}$ is in the pseudo-normal plane at $\gamma\left(s_{0}\right)$. Especially, if $\kappa\left(s_{0}\right) \neq 0$, then $\boldsymbol{v}_{0}=\boldsymbol{N}\left(\widehat{\left.s_{0}\right) \pm \boldsymbol{B}}\left(s_{0}\right)\right.$.

(2) $h^{\prime}\left(s_{0}\right)=h^{\prime \prime}\left(s_{0}\right)=0$ if and only if $\boldsymbol{v}_{0}=\boldsymbol{N}\left(\widetilde{\left.s_{0}\right) \pm \boldsymbol{B}}\left(s_{0}\right)=\widetilde{\boldsymbol{N}(s)}\right.$ and $\kappa\left(s_{0}\right)=0$. 
We consider the meaning of the above proposition as follows: Let $F:(\mathbb{R} \times \mathbb{R},(0,0)) \longrightarrow \mathbb{R}$ be a germ of smooth functions family. By the implicit function theorem, $\partial^{2} F / \partial t \partial u(0,0) \neq 0$ and $\partial F / \partial t(t, u)=0$ if and only if there exists a smooth function germ $g:(\mathbb{R}, 0) \longrightarrow(\mathbb{R}, 0)$ such that $u=g(t)$. Under this condition, $\partial^{2} F / \partial t^{2}(0,0)=0$ if and only if $g^{\prime}(0)=0$. Applying this general arguments to the lightcone height function family $H$, we have the following corollary:

Corollary 2.2 Let $\gamma: I \longrightarrow \mathbb{R}_{1}^{3}$ be a unit speed spacelike curve with $\gamma^{\prime \prime} \neq 0$. Then $s_{0} \in I$ is a singular point of the lightcone Gauss map (ie., $\left.\widetilde{\mathbb{L}}^{\prime}\left(s_{0}\right)=\mathbf{0}\right)$ if and only if $\kappa\left(s_{0}\right)=0$.

The main subject in this paper is a spacelike closed curve. Let $\gamma: S^{1} \longrightarrow \mathbb{R}_{1}^{3}$ be a smooth regular closed curve (i.e., $\dot{\gamma}(t) \neq 0$ ), where $S^{1}$ is a unit circle. Throughout in this paper, we assume that $S^{1}$ is oriented as the anti-clockwise direction. We say that $\gamma$ is spacelike if the tangent vector $\dot{\gamma}(t)$ is spacelike at any point $t \in S^{1}$. For any spacelike curve $\gamma(t)=$ $\left(x_{0}(t), x_{1}(t), x_{2}(t)\right)$, the relation $-\dot{x}_{0}(t)^{2}+\dot{x}_{1}(t)^{2}+\dot{x}_{2}(t)^{2}>0$ leads that $\pi \circ \dot{\gamma}(t)=\left(\dot{x}_{1}(t), \dot{x}_{2}(t)\right) \neq$ $\mathbf{0}$, so that $\pi \circ \boldsymbol{\gamma}$ is always a regular Euclidean plane curve. Moreover if a spacelike regular closed curve $\gamma$ is an embedding, we call it a spacelike knot.

\section{Spacelike regular homotopy}

In this section we consider regular homotopy classifications of regular spacelike closed curve. Let $\gamma_{0}, \gamma_{1}: S^{1} \longrightarrow \mathbb{R}_{1}^{3}$ be closed regular spacelike curves in $\mathbb{R}_{1}^{3}$. We say that $\gamma_{0}$ and $\gamma_{1}$ are spacelike regular homotopic if there exists a smooth map $F: S^{1} \times[0,1] \longrightarrow \mathbb{R}_{1}^{3}$ such that $F_{\tau}: S^{1} \longrightarrow \mathbb{R}_{1}^{3}$ is a closed regular spacelike curve at each $\tau \in[0,1]$ and $F_{0}=\gamma_{0}, F_{1}=\gamma_{1}$. Here, we denote $F_{\tau}(t)=F(t, \tau)$. By the argument in the last section, if $\gamma_{0}$ and $\gamma_{1}$ are spacelike regular homotopic, then $\pi \circ \gamma_{0}$ and $\pi \circ \gamma_{1}$ are regular homotopic as closed regular plane curves. It has been known that the regular homotopy classification among regular plane curves are classified by the rotation number [12]. Therefore the rotation number of the projection of a closed spacelike regular curve is a spacelike regular homotopy invariant. For regular spacelike closed curves, we have the following theorem:

Theorem 3.1 Let $\gamma_{0}, \gamma_{1}$ be regular spacelike closed curves. If $\pi \circ \gamma_{0}$ and $\pi \circ \gamma_{1}$ are regular homotopic as regular plane curves, then $\gamma_{0}$ and $\gamma_{1}$ are spacelike regular homotopic.

Proof. By the assumption, there exists a smooth mapping $H: S^{1} \times I \longrightarrow \mathbb{R}^{2}$ such that $H(t, 0)=$ $\gamma_{0}(t), H(t, 1)=\gamma_{1}(t)$ and $\partial H / \partial t(t, \tau) \neq \mathbf{0}$. We set $\gamma_{0}(t)=\left(x_{0}(t), x_{1}(t), x_{2}(t)\right)$. Since $\gamma_{0}$ is a spacelike curve, we have $-\dot{x}_{0}^{2}(t)+\dot{x}_{1}^{2}(t)+\dot{x}_{2}^{2}(t)>0$ and $\dot{x}_{1}^{2}(t)+\dot{x}_{2}^{2}(t)=\|\partial H / \partial t(t, 0)\|^{2}$. Therefore we have $\left|\dot{x}_{0}(t)\right|<\|\partial H / \partial t(t, 0)\|$. If we put $\gamma_{1}(t)=\left(y_{0}(t), y_{1}(t), y_{2}(t)\right)$, then we also have $\left|\dot{y}_{0}(t)\right|<$ $\|\partial H / \partial t(t, 1)\|$. Since $0<\|\partial H / \partial t(t, \tau)\|$ for any $(t, \tau) \in S^{1} \times I$, there exists a smooth function $h: S^{1} \times I \longrightarrow \mathbb{R}$ such that $h(t, 0)=\dot{x}_{0}(t), h(t, 1)=\dot{y}_{0}(t)$ and $|h(t, \tau)|<\|\partial H / \partial t(t, \tau)\|$. We now define a smooth mapping $F: S^{1} \times I \longrightarrow \mathbb{R}_{1}^{3} ; F(t, \tau)=\left(f_{0}(t, \tau), f_{1}(t, \tau), f_{2}(t, \tau)\right)$ where

$$
f_{0}(t, \tau)=\int_{0}^{t} h(s, \tau) d s+\tau y_{0}(0)+(1-\tau) x_{0}(0),\left(f_{1}(t, \tau), f_{2}(t, \tau)\right)=H(t, \tau) .
$$

Since $\partial f_{0} / \partial t(t, \tau)=h(t, \tau)$, we have

$$
\left(\frac{\partial f_{0}}{\partial t}(t, \tau)\right)^{2}<\left\|\frac{\partial H}{\partial t a}(t, \tau)\right\|^{2}=\left(\frac{\partial f_{1}}{\partial t}(t, \tau)\right)^{2}+\left(\frac{\partial f_{2}}{\partial t}(t, \tau)\right)^{2} .
$$


Moreover $\partial F / \partial t(t, \tau)=\left(\partial f_{0} / \partial t(t, \tau), \partial H / \partial(t, \tau)\right) \neq \mathbf{0}$. We also have $f_{0}(t, 0)=\int_{0}^{t} \dot{x}_{0}(s) d s+$ $x_{0}(0)=x_{0}(t)-x_{0}(0)+x_{0}(0)=x_{0}(t)$ and $f_{0}(t, 1)=\int_{0}^{t} \dot{y}_{0}(s) d s+y_{0}(0)=y_{0}(t)-y_{0}(0)+y_{0}(0)=$ $y_{0}(t)$. Therefore we have a spacelike regular homotopy $F$ between $\gamma_{0}$ and $\gamma_{1}$.

For a regular closed plane curve $\gamma: S^{1} \longrightarrow \mathbb{R}^{2}$, we denote the rotation number of $\gamma$ by $R(\gamma)$. We have the following corollary of the above theorem and Whitney's classification theorem:

Corollary 3.2 Let $\gamma_{0}$ and $\gamma_{1}$ be spacelike regular closed curve in $\mathbb{R}_{1}^{3}$. Then $\gamma_{0}$ and $\gamma_{1}$ are spacelike regular homotopic if and only if $R\left(\pi \circ \gamma_{0}\right)=R\left(\pi \circ \gamma_{1}\right)$.

We remark that the rotation number of the projection of a closed spacelike regular curve is a complete invariant under the spacelike regular homotopy.

On the other hand, we try to calculate the rotation number of a spacelike regular closed curve from the view point of Lorentzian differential geometry. Let $\gamma: S^{1} \longrightarrow \mathbb{R}_{1}^{3}$ be a unit speed closed spacelike regular curve with $\gamma^{\prime \prime} \neq 0$. We now fix the parameterization of the lightcone circle:

$$
S_{+}^{1}=\{(1, \cos \theta, \sin \theta) \mid 0 \leq \theta<2 \pi\} .
$$

It follows that we can denote that

$$
\widetilde{\mathbb{L}}(s)=(1, \cos \theta(s), \sin \theta(s))
$$

by a smooth function $\theta(s)$. Then we have the following proposition:

Proposition 3.3 Under the same notations as the above, we have the following relation:

$$
\frac{d \theta}{d s}(s)=\frac{-\delta(\gamma(s))}{\ell_{0}(s)} \kappa(s)
$$

at $s \in S^{1}$ with $\kappa(s) \neq 0$. If $\kappa(s)=0$ then $d \theta / d s(s)=0$.

Proof. Firstly we assume that $\kappa(s) \neq 0$. By definition, we have

$$
\widetilde{\mathbb{L}}^{\prime}(s)=\left(0,-\sin \theta(s) \frac{d \theta}{d s}(s), \cos \theta(s) \frac{d \theta}{d s}(s)\right) .
$$

If we write $\boldsymbol{n}(s)=\left(n_{0}(s), n_{1}(s), n_{2}(s)\right)$, then we calculate the following determinant:

$$
\begin{aligned}
\left|\boldsymbol{n}(s), \widetilde{\mathbb{L}}(s), \widetilde{\mathbb{L}}^{\prime}(s)\right| & =\left|\begin{array}{ccc}
n_{0}(s) & n_{1}(s) & n_{2}(s) \\
1 & \cos \theta(s) & \sin \theta(s) \\
0 & -\sin \theta(s)(d \theta / d s)(s) & \cos \theta(s)(d \theta / d s)(s)
\end{array}\right| \\
& =\frac{d \theta}{d s}(s)\left(n_{0}(s)-n_{1}(s) \cos \theta(s)-n_{2}(s) \sin \theta(s)\right) \\
& =-\frac{d \theta}{d s}(s)\langle\boldsymbol{n}(s), \widetilde{\mathbb{L}}(s)\rangle \\
& =\frac{d \theta}{d s}(s)\left\langle\boldsymbol{n}(s), \frac{1}{\ell_{0}(s)}(\boldsymbol{n}(s)+\boldsymbol{b}(s))\right\rangle \\
& =-\frac{\delta(\gamma(s))}{\ell_{0}(s)} \frac{d \theta}{d s}(s) .
\end{aligned}
$$


On the other hand, since $\mathbb{L}=\ell_{0} \tilde{\mathbb{L}}$, we have $\mathbb{L}^{\prime}=\ell_{0}^{\prime} \widetilde{\mathbb{L}}+\ell_{0} \widetilde{\mathbb{L}}^{\prime}$. Moreover, by the Frenet-Serret type formula, we have $\mathbb{L}^{\prime}=-\delta(\gamma) \kappa \boldsymbol{t}+\tau(\boldsymbol{n}+\boldsymbol{b})$. It follows that

$$
\begin{aligned}
\left|\boldsymbol{n}(s), \widetilde{\mathbb{L}}(s), \widetilde{\mathbb{L}}^{\prime}(s)\right| & =\left|\boldsymbol{n}(s), \frac{1}{\ell_{0}(s)} \mathbb{L}(s), \frac{1}{\ell_{0}(s)} \mathbb{L}^{\prime}(s)+\frac{\ell_{0}^{\prime}(s)}{\ell_{0}(s)} \mathbb{L}(s)\right| \\
& =\mid \boldsymbol{n}(s), \frac{1}{\ell_{0}(s)}(\boldsymbol{n}(s)+\boldsymbol{b}(s)), \frac{1}{\ell_{0}(s)}(-\delta(\gamma(s)) \kappa(s) \boldsymbol{t}(s)+\tau(s)(\boldsymbol{n}(s)+\boldsymbol{b}(s) \mid \\
& =\frac{-\delta(\gamma(s)) \kappa(s)}{\ell_{0}^{2}(s)}|\boldsymbol{n}(s), \boldsymbol{b}(s), \boldsymbol{t}(s)| \\
& =\frac{-\delta(\gamma(s)) \kappa(s)}{\ell_{0}^{2}(s)} \mid(-\delta(\gamma(s)))=\frac{\kappa(s)}{\ell_{0}^{2}(s)} .
\end{aligned}
$$

Therefore we have the desired relation.

By Corollary 2.2, $\kappa(s)=0$ if and only if $s$ is a singular point of the lightcone Gauss map. This is equivalent to the condition $d \theta / d s(s)=0$.

We define the normalized curvature $\bar{\kappa}(s)$ by

$$
\bar{\kappa}(s)=\frac{-\delta(\gamma(s))}{\ell_{0}(s)} \kappa(s)
$$

Then we have the following proposition:

Proposition 3.4 For any unit speed closed regular spacelike curve $\gamma: S^{1} \longrightarrow \mathbb{R}_{1}^{3}$ with $\gamma^{\prime \prime} \neq 0$, we have

$$
\frac{1}{2 \pi} \int_{S^{1}} \bar{\kappa}(s) d s=\operatorname{deg} \tilde{\mathbb{L}}
$$

where $\operatorname{deg} \widetilde{\mathbb{L}}$ is the mapping degree of $\widetilde{\mathbb{L}}$.

Proof. By Proposition 3.3, we have

$$
\int_{S^{1}} \bar{\kappa}(s) d s=\int_{0}^{2 \pi} \frac{d \theta}{d s}(s) d s=2 \pi \operatorname{deg} \tilde{\mathbb{L}} .
$$

By using the canonical projection $\pi:\left(\mathbb{R}^{3} \longrightarrow \mathbb{R}^{2}\right.$, we have an orientation preserving diffeomorphism $\pi \mid S_{+}^{1}: S_{+}^{1} \longrightarrow S^{1}$. We now consider the (Euclidean) Gauss map

$$
\mathbb{N}: S^{1} \longrightarrow S^{1}
$$

on $\pi \circ \gamma$.

We need the following lemma.

Lemma 3.5 For any unit speed closed regular spacelike curve $\gamma: S^{1} \longrightarrow \mathbb{R}_{1}^{3}$, the vector $\pi \circ \widetilde{\mathbb{L}}(s)$ is transverse to $(\pi \circ \gamma)^{\prime}(s)$ at any point $s \in S^{1}$. 
Proof. Suppose that there is a point $s \in S^{1}$ such that the vector $\pi \circ \widetilde{\mathbb{L}}(s)$ is not transverse to $(\pi \circ \gamma)^{\prime}(s)$. Since $\pi \circ \gamma$ is a regular curve in $\mathbb{R}^{2}$, we have $\pi \circ \widetilde{\mathbb{L}}(s) \in\left\langle(\pi \circ \gamma)^{\prime}(s)\right\rangle_{\mathbb{R}}$. Therefore we have

$$
\widetilde{\mathbb{L}}(s) \in\left\langle\gamma^{\prime}(s)\right\rangle_{\mathbb{R}}+\operatorname{Ker} d \pi_{\gamma(s)} .
$$

On the other hand, $\widetilde{\mathbb{L}}(s)$ is in the pseudo-normal plane of $\gamma$ at $s$ and $\widetilde{\mathbb{L}}(s) \notin \operatorname{Ker} d \pi_{\gamma(s)}$. Since Ker $d \pi_{\gamma(s)}$ is a timelike one-dimensional subspace in $T_{\gamma(s)} \mathbb{R}_{1}^{3}$, we have

$$
\left\langle\widetilde{\mathbb{L}}(s), \operatorname{Ker} d \pi_{\gamma(s)}\right\rangle_{\mathbb{R}}+\left\langle\gamma^{\prime}(s)\right\rangle_{\mathbb{R}}=T_{\gamma(s)} \mathbb{R}_{1}^{3} .
$$

However, by the assumption, the dimension of the vector space in the left hand side is at most 2. This is a contradiction.

Lemma 3.6 Under the choice of a suitable direction of $\mathbb{N}, \pi \circ \widetilde{\mathbb{L}}$ and $\mathbb{N}$ are homotopic.

Proof. Since $\widetilde{\mathbb{L}}$ is transverse to $\pi \circ \gamma\left(S^{1}\right)$ in $\mathbb{R}^{2},\langle\pi \circ \widetilde{\mathbb{L}}(s), \mathbb{N}(s)\rangle \neq 0$ at any $s \in S^{1}$. Since $S^{1}$ is connected, we choose the direction of $\mathbb{N}$ such that makes $\langle\pi \circ \widetilde{\mathbb{L}}(s), \mathbb{N}(s)\rangle>0$.

We now construct a homotopy between $\pi \circ \widetilde{\mathbb{L}}$ and $\mathbb{N}$. Let

$$
F: S^{1} \times[0,1] \longrightarrow S^{1}
$$

be defined by

$$
F(s, t)=\frac{t \mathbb{N}(s)+(1-t) \pi \circ \widetilde{\mathbb{L}}(s)}{\|t \mathbb{N}(s)+(1-t) \pi \circ \widetilde{\mathbb{L}}(s)\|},
$$

where $\|\cdot\|$ is the Euclidean norm.

If there exists $t^{\prime} \in[0,1]$ and $s^{\prime} \in S^{1}$ such that $t^{\prime} \mathbb{N}\left(s^{\prime}\right)+\left(1-t^{\prime}\right) \pi \circ \widetilde{\mathbb{L}}\left(s^{\prime}\right)=\mathbf{0}$, then we have $\mathbb{N}\left(s^{\prime}\right)=-\pi \circ \widetilde{\mathbb{L}}\left(s^{\prime}\right)$. This contradicts to the assumption that $\langle\pi \circ \widetilde{\mathbb{L}}, \mathbb{N}(s)\rangle>0$. Therefore $F$ is a continuous mapping satisfying $F(s, 0)=\pi \circ \tilde{\mathbb{L}}(s)$ and $F(s, 1)=\mathbb{N}(s)$ for any $s \in S^{1}$.

Since the mapping degree is a homotopy invariant and a invariant under orientation preserving diffeomorphisms, we have the following corollary.

Corollary 3.7 Under the same assumptions as those in Proposition 3.4, we have

$$
\operatorname{deg} \widetilde{\mathbb{L}}=R(\pi(\gamma))
$$

By the definition of the normalized lightcone curvature $\bar{\kappa}$, we obtain:

$$
\frac{1}{2 \pi} \int_{S^{1}} \bar{\kappa}(s) d s=\operatorname{deg}(\widetilde{\mathbb{L}})=R(\pi(\gamma)) .
$$

This proves the following theorem.

Theorem 3.8 For any unit speed closed regular spacelike curve $\gamma: S^{1} \longrightarrow \mathbb{R}_{1}^{3}$ with $\gamma^{\prime \prime} \neq 0$, we have

$$
\frac{1}{2 \pi} \int_{S^{1}} \bar{\kappa}(s) d s=R(\pi(\gamma))
$$




\section{Spacelike knots}

In this section we consider spacelike knots in Minkowski 3-space. This section depends heavily on results in the ordinary knot theory in Euclidean 3-space. For fundamental concepts, please refer [9].

Although the notion of spacelike knots is defined as smooth spacelike embeddings, we consider in the PL-category like as the ordinary knot theory. A spacelike knot $K$ is defined to be a closed polygon in Minkowski 3-space satisfying the following conditions:

(1) Any age of $K$ is a part of a spacelike line.

(2) For any age $A B$, the projection image $\pi(A B)$ is also a line segment in the Euclidean plane.

(2) If we have two adjacent edges $A B, B C$ of $K$, then $\pi(A B) \cap \pi(B C)=\{\pi(B)\}$.

By the original definition of spacelike curves in the smooth category, the projection image of a spacelike curve is a regular curve in the Euclidean plane. Therefore we need the condition (2) and (3) in the PL-category.

On the given spacelike knot $K$ we may perform the following four operations:

(1) We may divide an edge, $A B$, in space of $K$ into two edges, $A C, C B$, by placing a point $C$ on the edge.

(2) [The converse of (1)] If $A C$ and $C B$ are two adjacent edges of $K$ such that if $C$ is erased $A B$ becomes a straight line, then we may remove the point $C$.

(3) Suppose $C$ is a point in space that does not lie on $K$. Consider edges $X A, A B, B Y$ in $K$. If $A C, C B$ are parts of spacelike lines and the triangle $\triangle A B C$, formed by $A B$ and $C$, does not intersect $K$, with the exception of the edge $A B$, moreover $\pi(X A) \cap \pi(\triangle A B C)=$ $\pi(B Y) \cap \pi(\triangle A B C)=\emptyset$, we may remove $A B$ and add the two edges $A C$ and $C B$.

(4) [The converse of (3)] If there exists in space a triangle $\triangle A B C$ that contains two adjacent edges $A C$ and $C B$ of $K$, and this triangle does not intersect $K$, except at the edges $A C$ and $C B$, moreover $\pi(X A) \cap \pi(\triangle A B C)=\pi(B Y) \cap \pi(\triangle A B C)=\emptyset$, then we may delete the two edges $A C, C B$ and add the edge $A B$.

These four operations (1), (2), (3), (4) are called the elementary spacelike knot move. A spacelike knot $K$ is said to be spacelike equivalent (or,spacelike isotopic) to a spacelike knot $K^{\prime}$ if we obtain $K^{\prime}$ by applying the elementary spacelike knot moves a finite number of times. We can also consider the orientation of spacelike knots as usual.

We consider the projection $\pi(K)$ of a spacelike knot $K$. Like as the ordinary knot projection, by performing several elementary spacelike knot moves, we can impose the following conditions:

(1) $\pi(K)$ has at most a finite number of points of intersection.

(2) If $Q$ is a point of intersection of $\pi(K)$, then the inverse image, $\pi^{-1}(Q) \cap K$, of $Q$ in $K$ has exactly two points. That is, $Q$ is a double point of $\pi(K)$.

(3) A vertex of $K$ is never mapped onto a double point of $\pi(K)$.

The projection $\pi(K)$ that satisfies the above conditions is said to be a Minkowski spacelike regular projection. If we distinct the knot passes over or under itself at each double point, we have a Minkowski spacelike regular diagram.

We now consider the analogy of the Reidemeister moves of ordinary knots. In the ordinary knot theory, there are three kinds of moves $\Omega_{1}, \Omega_{2}$ and $\Omega_{3}$ and/or their inverses which are called the Reidemeister moves. Of course, we ignore the trivial moves $\Omega^{*}, \Omega_{0}$ here. We now call just the two moves $\Omega_{2}$ and $\Omega_{3}$ and/or their inverse the spacelike Reidemeister moves. We can 
easily show that all spacelike Reidemeister moves can be obtained as sequences of elementary spacelike moves.
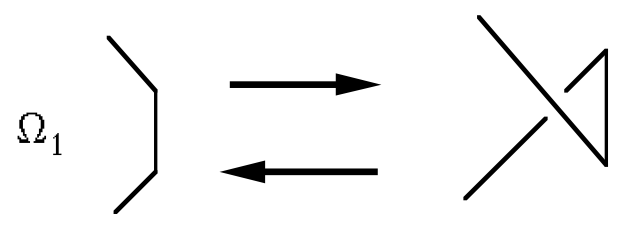

or
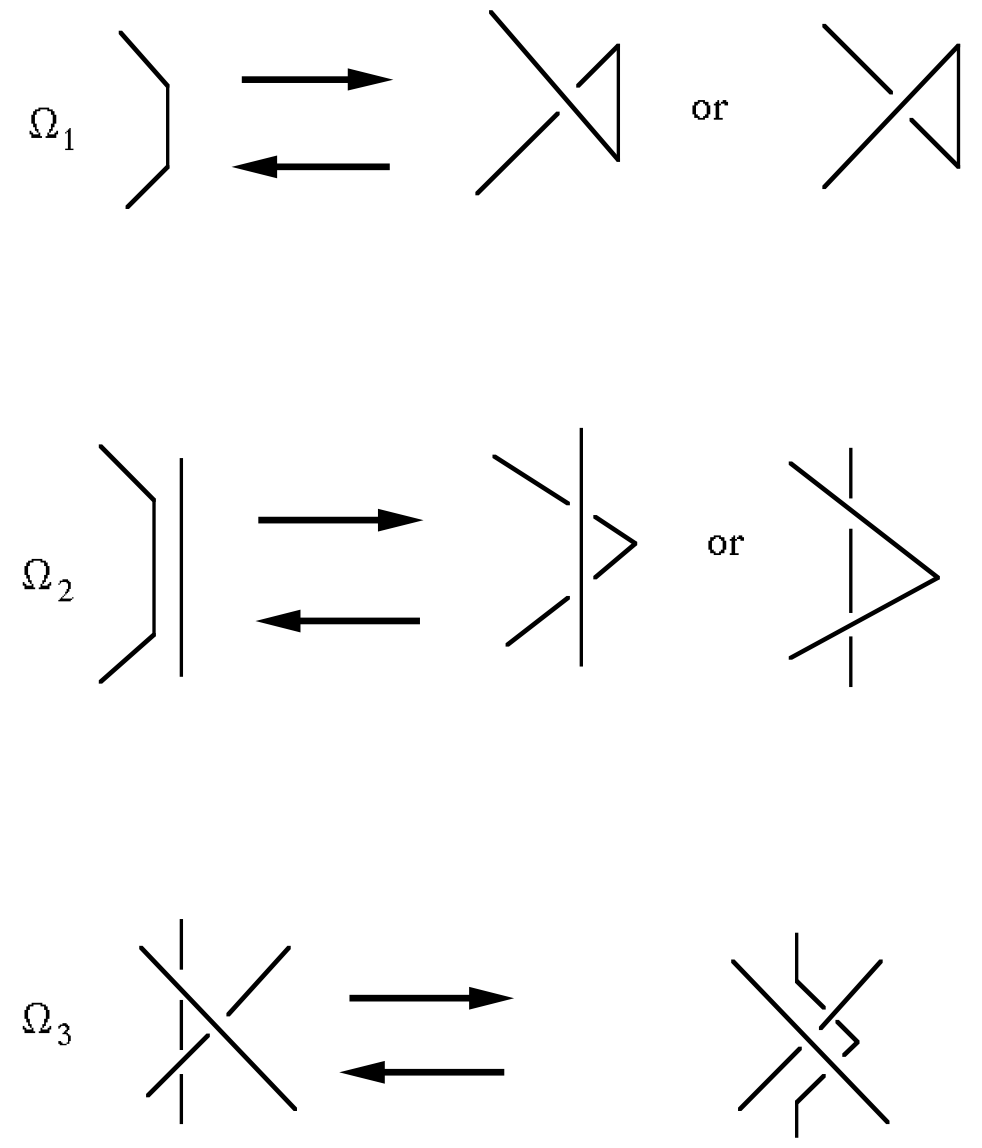

Figure 1: The Reidemeister moves

Let $K$ and $K^{\prime}$ be spacelike knots with Minkowski spacelike regular diagrams $\pi(K)$ and $\pi\left(K^{\prime}\right)$, respectively. Then $\pi(K)$ and $\pi\left(K^{\prime}\right)$ are equivalent as Minkowski spacelike regular diagrams (or briefly, $M$-s-equivalent) if we can change $\pi(K)$ to $\pi\left(K^{\prime}\right)$ by performing, a finite number of times, the spacelike Reidemeister moves (with trivial moves). We remark that Ms-equivalent has been called the regular isotopy among regular diagrams in the ordinary knot theory. We can show the following theorem:

Theorem 4.1 Let $K$ and $K^{\prime}$ be spacelike knots with Minkowski spacelike regular diagrams $\pi(K)$ and $\pi\left(K^{\prime}\right)$, respectively. Then $K$ and $K^{\prime}$ are spacelike equivalent if and only if $\pi(K)$ and $\pi\left(K^{\prime}\right)$ are $M$-s-equivalent.

The proof of Theorem 4.1 is almost parallel to that of the corresponding theorem in the ordinary knot theory (cf., [9], Theorem 4.1.1). We can verify that we do not use the $\Omega_{1}$ move and its inverse for each step of the proof in the ordinary knot theory. Therefore we only describe the outline of the proof here. For simplicity, we call a finite number of times a composition of spacelike Reidemeister moves a spacelike $R$-move. 
We can easily show that the following 7 moves are spacelike R-moves:

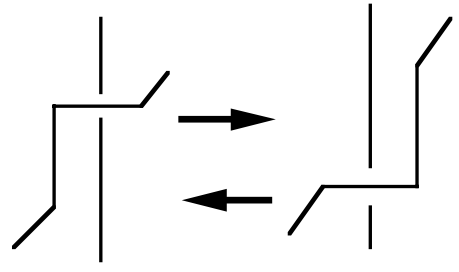

(a1)
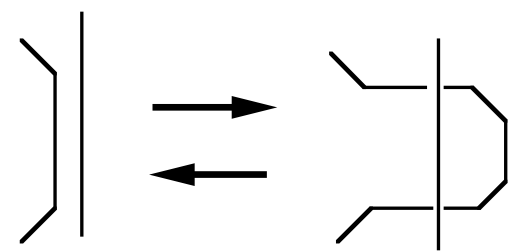

(b1)

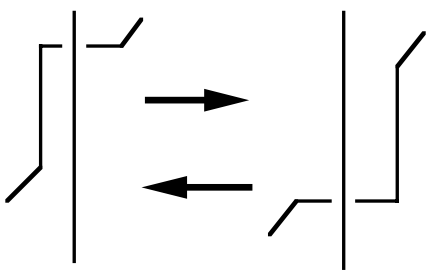

(a2) or

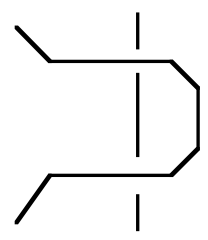

(b2)

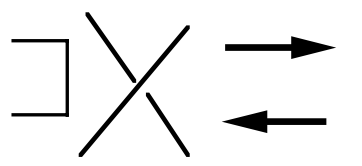

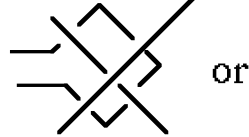

(c1)

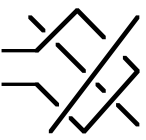

(c2)

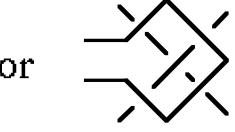

(c3)

Figure 2: $a 1, a 2, b 1, b 2, c 1, c 2, c 3$

Outline of the proof of Theorem 4.1. Let $K$ be a spacelike knot. Suppose that $K^{\prime}$ is obtained by replacing $A B$ of $K$ by two edges $A C \cup C B$ of $\triangle A B C$. Here we write $a=\pi(A), b=$ $\pi(B), c=\pi(B)$ etc. In the ordinary knot theory we need to consider two cases in Fig. 3. However, the case (b) cannot be occurred as an elementary spacelike knot moves, so that we only consider the case (a). We denote that $\pi(\triangle A B C)=\triangle a b c$ and $\pi(A B)=a b$. Therefore, it is enough to show that we can change $W=a c \cup c b$ to the segment $a b$ by repeatedly using spacelike R-moves. In the proof of ([9], Theorem 4.1.1), we can verify that only the above spacelike R-moves $\{a 1, a 2, b 1, b 2, c 1, c 2 . c 3\}$ are used. Therefore we change $\pi(K)$ to $\pi\left(K^{\prime}\right)$ by performing, a finite number of times, the spacelike Reidemeister moves.

The equivalence relation on regular diagrams generated by the Reidemeister moves $\Omega_{2}$ and $\Omega_{3}$ has been called a regular isotopy [6]. Therefore Theorem 4.1 asserts that $K$ and $K^{\prime}$ are spacelike isotopic if and only if $\pi(K)$ and $\pi\left(K^{\prime}\right)$ are regular isotopic. We now consider regular isotopic invariants for regular diagrams. We consider oriented knots. Let assign either +1 or -1 to each crossing point of a regular diagram of an oriented knot. At a crossing point, $c$, of an oriented spacelike regular diagram, as shown in Fig. 4, we have two possible configurations. 


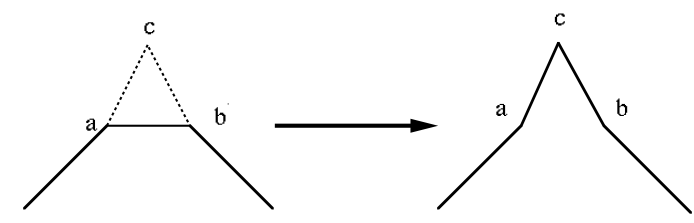

(a)

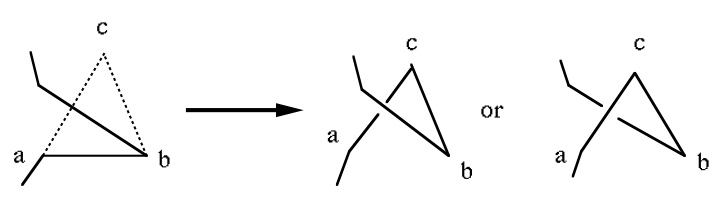

(b)

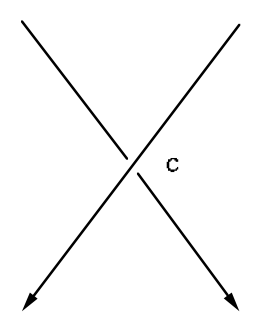

$\operatorname{sign}(\mathrm{c})=+1$

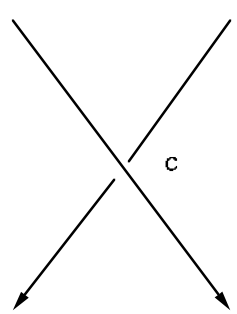

$\operatorname{sign}(\mathrm{c})=-1$

Figure 4: Signatures

Figure 3: Two cases for the ordinary knot

In case (a) we assign $\operatorname{sign}(c)=+1$ to the crossing point, while in case (b) we assign $\operatorname{sign}(c)=-1$. For an oriented spacelike knot $K$, we have the oriented Minkowski spacelike regular diagram $\pi(K)$. Then, the sum $w(K)$ of the signs of all the crossing points of $\pi(K)$ is said to be the writhe (or, the Tait number) of $K$. In [10], it has been shown the following theorem:

Theorem 4.2 Let $K$ and $K^{\prime}$ be oriented knots in Euclidean space with the oriented regular diagrams $D$ and $D^{\prime}$ of a fixed regular projection respectively. Then $D$ is regular isotopic to $D^{\prime}$ if and only if $K$ is isotopic to $K^{\prime}$ and $(R(D), w(D))=(R(D), w(D))$.

As a corollary of Theorems 4.1 and 4.2, we have the following classification theorem:

Theorem 4.3 Let $K$ and $K^{\prime}$ be oriented spacelike knots. Then $K$ is spacelike isotopic to $K^{\prime}$ if and only if $K$ is isotopic to $K^{\prime}$ (as ordinary knots) and

$$
(R(\pi(K)), w(\pi(K)))=\left(R\left(\pi\left(K^{\prime}\right)\right), w\left(\pi\left(K^{\prime}\right)\right)\right) .
$$

\section{References}

[1] S. Izumiya and A. Takiyama, A time-like surface in Minkowski 3-space which contains pseudocircles, Proc. Edinburgh Math. Soc. (2) 40 (1997), 127-136

[2] S.Izumiya, D.Pei and T.Sano, The lightcone Gauss map and the lightcone developable of a spacelike curve in Minkowski 3-space, Glasgow.Math.J., vol 42 (2000), 75-89 
[3] S. Izumiya, D. Pei and M.C. Romero Fuster, The lightcone Gauss map of a spacelike surface in Minkowski 4-space, Asian J. Math., vol. 8 (2004), 511-530

[4] S. Izumiya, M. Kossowski, D. Pei and M.C. Romero Fuster, Singularities of lightlike hypersurfaces in Minkowski 4-space, preprint

[5] S. Izumiya, D. Pei and M.C. Romero Fuster, Umbilicity of spacelike submanifolds of Minkowski space, Proceedings of the Royal Society of Edinburgh, 134A (2004), 375-387

[6] L. Kauffman, An invariant of regular isotopy, Transaction of the American Mathematical Society, vol. 318 (1990), 417-471

[7] M. Kossowski, The $S^{2}$-valued Gauss maps and split total cuvature of space-likecodimension-2 surface in Minkowski space, J. London Math. Soc.(2) vol 40, (1989),179-192

[8] M. Kossowski, The intrinsic conformal structure and Gauss map of a light-like hypersurface in Minkowski space, Transcations of the American mathematical society vol 316.Number 1, (1989), 369-383

[9] K. Murasugi, Knot Theory and Its Applications, Birkhäuser, Boston·Basel-Berlin (1996)

[10] B. Trace, On the Reidemeister moves of a classical knot, Proc. of American Math. Soc., vol 89 (1983), 722-724

[11] B. O’Neill, Semi-Riemannian Geometry, Academic Press, New York (1983)

[12] H.Whitney, On regular closed curves in the plane, Compositio Math., 4, (1937), 276-284

Shyuichi Izumiya, Department of Mathematics, Hokkaido University, Sapporo 060-0810,Japan e-mail:izumiya@math.sci.hokudai.ac.jp

Makoto Kikuchi, Ritsumeikan Keisho Junior and Senior High School, Ebetsu 069-0823,Japan

Masatomo Takahashi, Department of Mathematics, Hokkaido University, Sapporo 060-0810,Japan e-mail:takahashi@math.sci.hokudai.ac.jp 